\title{
Time-resolution of the shoot and root growth of the model cereal Brachypodium in response to inoculation with Azospirillum bacteria at low phosphorus and temperature
}

\author{
Martino Schillaci $^{1}$ (D) $\cdot$ Borjana Arsova $^{2}$ (D) $\cdot$ Robert Walker $^{1}$ (D) Penelope M. C. Smith $^{3}$ (D) Kerstin A. Nagel $^{2}$ (D) \\ Ute Roessner $^{1}$ D . Michelle Watt ${ }^{1}$ (D)
}

Received: 22 May 2020 / Accepted: 20 October 2020 / Published online: 1 November 2020

(c) The Author(s) 2020

\begin{abstract}
A non-invasive plant phenotyping platform, GrowScreen-PaGe, was used to resolve the dynamics of shoot and root growth of the model cereal Brachypodium (Brachypodium distachyon Bd21-3) in response to the plant growth promoting (PGP) bacteria Azospirillum (Azospirillum brasilense Sp245). Inoculated Brachypodium plants had greater early vigor and higher $\mathrm{P}$ use efficiency than non-inoculated Brachypodium at low $\mathrm{P}$ and low temperature conditions. Root systems were imaged non-invasively at eight time points and data combined with leaf area, shoot biomass and nutrient content from destructive subsamples at 7, 14 and 21 days after inoculation (DAI). Azospirillum colonisation of roots improved Brachypodium shoot and, to a greater degree, root growth in three independent experiments. Inoculation promoted $\mathrm{P}$ use efficiency in shoots but not $\mathrm{P}$ concentration or uptake, despite increased total root length. Longer roots in inoculated plants arose from twofold faster branch root growth but slower axile root growth, detected at 11 DAI. Analysis of the spatio-temporal phenotypes indicated that the effects of Azospirillum inoculation increased as shoot $\mathrm{P}$ concentration declined, but the magnitude depended on the time after inoculation and growth rate of branch roots compared to axile roots. High throughput plant phenotyping platforms allow the details of plant-microorganism symbioses to be resolved, offering insights into the timing of changes in different tissues to allow molecular mechanisms to be determined.
\end{abstract}

Keywords Plant growth promoting (PGP) bacteria $\cdot$ Cereals $\cdot$ Phenotyping $\cdot$ Root architecture $\cdot$ Brachypodium distachyon Bd21-3 · Azospirillum brasilense Sp245

Electronic supplementary material The online version of this article (https://doi.org/10.1007/s10725-020-00675-4) contains supplementary material, which is available to authorized users.

Martino Schillaci

mschillaci@student.unimelb.edu.au

1 University of Melbourne, School of BioSciences, Parkville, VIC 3010, Australia

2 Institute for Bio- \& Geosciences, Plant Sciences (IBG-2), Forschungszentrum Juelich GmbH, 52425 Juelich, Germany

3 School of Life Sciences, Latrobe University, Department of Animal, Plant, and Soil Sciences, Bundoora, VIC 3086, Australia

\section{Introduction}

Cereals are consumed in all societies and retain a leading position in diets by providing almost one half of the calories consumed by humans (FAO 2018). Increasing agricultural production while availability of arable land decreases is one of the largest challenges facing modern agriculture, particularly for cereal crops. Good soil fertility depends on availability of phosphorus $(\mathrm{P})$ for crop growth. $\mathrm{P}$ is essential for a multitude of plant processes and is a significant limitation to production in many areas of the world due to decreasing availability of rock mineral phosphates, leading to increasing market price (Cordell et al. 2011). Phosphorus use efficiency by cereals is typically low, with a large proportion of $\mathrm{P}$ applied to a crop as fertilizer creating bonds with other elements such as $\mathrm{Ca}, \mathrm{Fe}$ and $\mathrm{Al}$, hence becoming unavailable for plant uptake (Dhillon et al. 2017). Many cereals germinate in winter, and the early stages of their growth often are 
subjected to suboptimal temperatures, which can negatively affect the plant nutrient uptake capacity (Grant et al. 2001; Hanway and Olson 1980) and inhibit root system elongation (McMichael and Quisenberry 1993). Low P conditions are typical of many cereal agricultural systems subject to cool temperature, causing poor early growth and seedling vigor after sowing in spring or autumn. Early plant vigor is a critical trait for capturing fertilizer and water soon after planting (Palta and Watt 2009) and is positively correlated with yield of cereals in many environments (Richards et al. 2007).

Numerous studies have shown that soil beneficial bacteria, so-called plant growth promoting (PGP) bacteria, can improve early plant vigor (Biswas et al. 2000; Gholami et al. 2009). We are not aware, however, of published results on the effects of PGP bacteria on plant seedling vigor when subjected to low $\mathrm{P}$ and temperature together. Indeed, studies on the combined effects of these stresses on cereals are scarce and quite dated (Baon et al. 1994; Batten et al. 1986). We have therefore investigated the interaction between the well-studied PGP bacteria strain Azospirillum brasilense Sp245 (Rothballer et al. 2005) and the model grass Brachypodium distachyon $\mathrm{Bd} 21-3$ (Catalan et al. 2014) in these conditions. A. brasilense Sp245 can promote plant growth, notably root length, through the production of PGP hormones (Dobbelaere et al. 1999), and this has been observed mainly when plants grow in suboptimal conditions (da Fonseca Breda et al. 2019; Pereyra et al. 2009). The response of B. distachyon root systems to low $\mathrm{P}$ depends on genotype, substrate, degree and duration of P deficiency (Ingram et al. 2012; Poiré et al. 2014; Sasse et al. 2019). Responses to root-associated microorganisms also vary (Do Amaral et al. 2016; Fitzgerald et al. 2015; Gagne-Bourque et al. 2015; Schneebeli et al. 2016). In previous studies on the interaction between $B$. distachyon and A. brasilense, Delaplace et al. (2015) exposed plant roots to bacterial volatiles and grew them on nutrient rich agar, and Do Amaral et al. (2016) studied the effects of the inoculation with both $A$. brasilense FP2-7 and Herbaspirillum seropedicae RAM4 on $B$. distachyon genotypes grown under no or low $\mathrm{N}$ conditions in soil. To our knowledge no genotype of B. distachyon has been tested for its response to any strain of $A$. brasilense under low $\mathrm{P}$ and temperature conditions. In the present study, we tested the hypothesis that Brachypodium distachyon $\mathrm{Bd} 21-3$ inoculated with $A$. brasilense $\mathrm{Sp} 245$ would have greater vigor than non-inoculated plants in low $\mathrm{P}$ and low temperature conditions, and that this would result from differences in the timing of shoot and root responses to inoculation. We used a phenotyping platform to non-invasively measure the same plants over successive time points to test our hypothesis.

Investigations of early interactions between plant roots and microorganisms have used destructive methods in the past. For instance, Casanovas et al. (2002) measured the effect of bacterial inoculation on water stressed maize seedlings 15 days after planting. Egamberdieva (2009) recorded germination and growth of seeds in saline conditions with or without inoculation at five days. Noninvasive imaging has been used to quantify the effects of beneficial bacteria in high throughput systems at one time point (Wintermans et al. 2016). Here we used the GrowScreen-PaGe (Gioia et al. 2016) phenotyping platform to quantify the dynamics of plant-root microorganism interactions at multiple time points. This platform is a 2D image-based method specifically designed for high throughput and non-destructive measurements of plant roots at various stages of their growth. Non-destructive root phenotyping in soil is performed with $\mathrm{X}$ ray $\mathrm{CT}$ and magnetic resonance imaging (reviewed in Wasson et al. (2020)). However the throughput and optical resolutions of these approaches so far have been limited to smaller experiments with crops like barley (Flavel et al. 2012) and maize (Pflugfelder et al. 2017) that have thick branch roots relative to those of Brachypodium.

This study aims to quantify how the root-colonizing bacteria A. brasilense Sp245 modulated the growth of Brachypodium distachyon $\mathrm{Bd} 21-3$ under unfavorable growth conditions of low $\mathrm{P}$ and low temperatures with non-destructive phenotyping and destructive shoot growth and nutrient analyses. By providing insights into the tissue- and time-specific effects of the inoculation, we hope to advance knowledge of how PGP bacteria can improve growth of cereal crops in soils with low fertility and cool temperature.

\section{Materials and methods}

\section{Seeds sterilization and germination}

Brachypodium distachyon Bd21-3 (hereafter referred to as Brachypodium in this study) seeds were dehusked, sterilized as described by Sasse et al. (2019) and stored in Milli-Q water at $4{ }^{\circ} \mathrm{C}$ for $7 \mathrm{~d}$ to synchronize germination. Operating in a biosafety cabinet, seeds were then put between two layers of moist Grade 1 Qualitative Filter Paper (Whatman, Maidstone, UK) with embryos facing downwards in Petri dishes (Fig. 1a) that were then sealed and covered with foil to prevent light reaching the emerging roots. The plates were placed vertically to ensure roots emerged with gravity during germination and left to germinate for $48 \mathrm{~h}$ at room temperature $\left(21^{\circ} \mathrm{C}\right)$ in the dark. After this step, seedlings with a primary root of approximately $1.5 \mathrm{~cm}$ were selected for bacterial inoculation (inoculated plants) or mock-inoculation (non-inoculated) before transferring to the phenotyping system. 


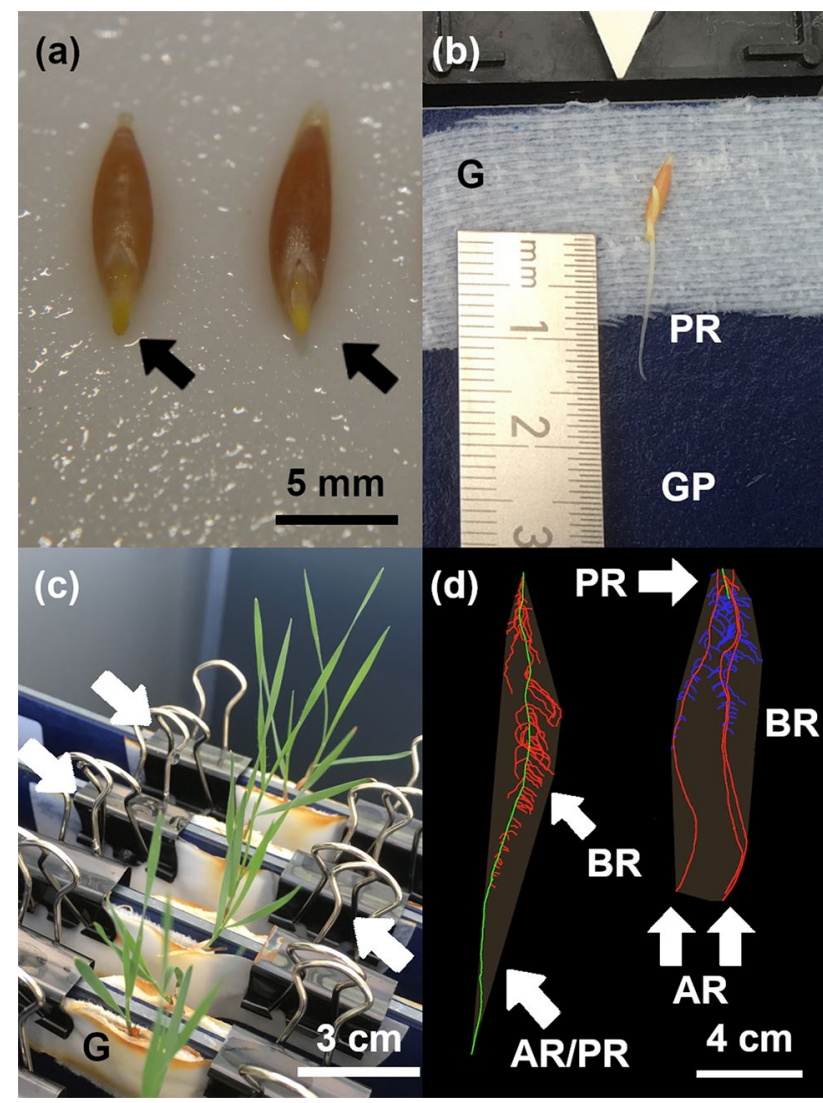

Fig. 1 Conditions for growth and phenotyping of Brachypodium with or without inoculation with Azospirillum in the GrowScreen-PaGe phenotyping platform. (a) Dehusked Brachypodium seeds were first placed on moist filter paper to synchronize germination at $4{ }^{\circ} \mathrm{C}$ for 7 days. Black arrows point to tips of primary axile roots (PR) emerging from the embryo at the base of the seeds. (b) Brachypodium seedling with $1.5 \mathrm{~cm}$ long PR on moist gauze $(\mathrm{G})$ on blue germination paper (GP) after inoculation with Azospirillum. (c) Brachypodium plants with 2-3 leaves mounted on GrowScreen-PaGe PVC plates with metal clips (white arrows). (d) Brachypodium root systems imaged 21 days after inoculation and after manual color-coding with PaintRhizo image analysis software according to root type. Left root system is typical of plants with the PR (green line) extending vertically through the experiment, becoming an axile root (AR) with branch roots (BR, red). Right root system is typical of plants whereby the PR stopped growing close to the seed (arrow), and new AR (red) extending vertically with $\mathrm{BR}$ (blue). PR primary axile root, $\mathrm{AR}$ axile root, BR branch root, G gauze, GP germination paper

\section{Bacterial inoculation}

Azospirillum brasilense Sp245 (hereafter referred to a Azospirillum in this study) (Rothballer et al. 2005) were maintained on lysogeny broth (LB) (Bertani 1951) with $1.2 \% \mathrm{w} / \mathrm{v}$ agar at room temperature prior to inoculation. The bacterial suspension for seedling inoculation was prepared by aliquoting a single bacterial colony from an agar plate into liquid $\mathrm{LB}$, and incubated overnight in a shaking incubator set at $210 \mathrm{rpm}$ and $28^{\circ} \mathrm{C}$. When the culture reached an OD600 of 0.8 (equivalent to $10^{7} \mathrm{CFUs} \mathrm{mL}^{-1}$ ), it was diluted with sterile phosphate buffered saline (PBS) to give a concentration of $10^{5} \mathrm{CFUs} \mathrm{mL}^{-1}$. Entire $48 \mathrm{~h}$ old Brachypodium seedlings germinated as described above were transferred to the bacterial culture for $90 \mathrm{~min}$ prior to transplanting to the plant phenotyping system. Non-inoculated plants were mock-inoculated in sterile PBS.

\section{Plant growth conditions}

Plants were phenotyped in the GrowScreen-PaGe system, a plant phenotyping platform originally designed to image root growth and architecture non-invasively over time at low or high throughput and under abiotic conditions such as nutrient deficiency (Gioia et al. 2016). Since this was the first study to use GrowScreen-PaGe with a biotic treatment, we conducted two low throughput pilot experiments to establish plant growth and measurement conditions. These were followed by one large high throughput experiment to allow quantification of the dynamics of growth. In these three experiments, plants are grown in the GrowScreen$P a G e$ system as described by Gioia et al. (2016) with the exception that $37.5 \times 23 \mathrm{~cm}$ polyvinyl chloride $(\mathrm{PVC}$ ) plates (Max Wirth GmbH, Braunschweig, DE) were used and steps were taken to minimize contamination and maintain experimental conditions for the inoculated seedlings (details below and see Fig. 1).

\section{First pilot experiment: Establishing growth and $P$ conditions in GrowScreen-PaGe}

A first pilot experiment was designed to test the GrowScreen$\mathrm{PaGe}$ for biotic phenotyping and to establish the $\mathrm{P}$ conditions where Azospirillum influenced Brachypodium growth at low temperature when compared with control (non-inoculated) plants grown at the same conditions. Non-inoculated and inoculated plants were provided with moderately low $\left(25 \mu \mathrm{M} \mathrm{KH}_{2} \mathrm{PO}_{4}\right)$ or extremely low $\left(7 \mu \mathrm{M} \mathrm{KH}_{2} \mathrm{PO}_{4}\right) \mathrm{P}$ levels. Twenty plants were grown for 21 days in each of the four conditions.

Working on a surface-sterilized bench, inoculated seedlings were transplanted to the GrowScreen-PaGe germination paper using sterile forceps. Seedlings were placed at the top of the paper and wrapped between two layers of moist, sterile cotton gauze (Fig. 1b, c) to protect the roots from light and dehydration. Following the results obtained by Gioia et al. (2016), dark blue 194 grade paper, $430 \mathrm{~g} \mathrm{~m}^{-2}$ (Ahlstrom Germany GmbH, Bärenstein, DE) was used as germination paper. The paper had been autoclaved and moistened with the nutrient solution described below, and attached to the PVC plates, such that each plate had a germination paper with a plant on both sides when placed vertically into the growth box. PVC plates and 
growth boxes had also been disinfected as described by Gioia et al. (2016) to minimize background microorganisms. Once in the growth boxes, the base of each germination paper was in nutrient solution that reached plant root systems by capillarity.

A total of four boxes were used, and each box contained plants subjected to a different condition. Each box had 12 plates, with two seedlings per plate (except the outer two plates, which were left empty to counter edge effects). The top of each box was covered with aluminum foil, leaving only a small opening for the shoot to grow, to prevent light from reaching the roots, reduce the evaporation rate of the nutrient solution and minimize contamination of the plates within the inoculated and control boxes. The GrowScreen$P a G e$ growth boxes were moved to a custom made climate chamber set at the day/night temperature of $20 / 10{ }^{\circ} \mathrm{C}$ and $70 \%$ relative air humidity with a $16 / 8 \mathrm{~h}$ light/dark cycle and light intensity of $250 \mu \mathrm{mol} \mathrm{m}{ }^{-2} \mathrm{~s}^{-1}$, provided by alternating $400 \mathrm{~W}$ HPI and SON-T lamps (Philips, Amsterdam, $\mathrm{NL}$ ) every $60 \mathrm{~min} .20 / 10^{\circ} \mathrm{C}$ is a cool temperature range for Brachypodium, which has an optimal growth temperature of $24 / 18{ }^{\circ} \mathrm{C}$ day/night (Harsant et al. 2013). The position of plants inside the boxes and boxes inside the chamber were rotated during the experiment every time roots were imaged.

Previous studies report that the optimal P substrate concentration for Brachypodium's growth is approximately $1.5 \mathrm{mM}$ in soil (Zhang et al. 2018) and $0.6 \mathrm{mM}$ in hydroponic systems (Poiré et al. 2014), respectively. Through the experiment, each box was supplied with $12 \mathrm{~L}$ of $1 / 3$ strength Hoagland solution (Hoagland and Arnon 1950), where $\mathrm{KH}_{2} \mathrm{PO}_{4}$ was supplied at a concentration of $7 \mu \mathrm{M}$ for the extremely low $\mathrm{P}$ treatment and $25 \mu \mathrm{M}$ for the moderately low $\mathrm{P}$ treatment. The solution was replaced weekly to prevent the depletion of nutrients (other than $\mathrm{P}$ ), excessive $\mathrm{pH}$ fluctuations and to decrease the risk of contamination (Gioia et al. 2016). All stock solutions were autoclaved before use and the working 1/3 Hoagland was diluted using deionized water.

\section{Second pilot experiment to reproduce and validate the first pilot experiment}

A second pilot experiment was done to verify the reproducibility and validity of the results observed in inoculated plants when compared with control (non-inoculated) plants at extreme low $\mathrm{P}$ availability $(7 \mu \mathrm{M} \mathrm{P})$ during the first pilot experiment. Seeds were sterilized, germinated, and seedlings were inoculated and grown as described above, with the exception that plants were inoculated with bacteria or noninoculated and grown with $7 \mu \mathrm{M} \mathrm{KH}_{2} \mathrm{PO}_{4}$. Twenty plants per treatment were grown for $21 \mathrm{~d}$ in the GrowScreen-PaGe.

\section{High throughput experiment}

Once the conditions were refined and the plant response to inoculation at extremely low $\mathrm{P}$ levels was confirmed in the pilot experiments, a third experiment was conducted in the GrowScreen-PaGe at much higher throughput and with higher sampling resolution to determine the impact of Azospirillum on shoot and root growth and nutrient concentrations in Brachypodium throughout development.

A total of 368 plants were divided into eight boxes, four containing inoculated plants and four containing control (non-inoculated) plants. Each box had 25 PVC plates with two seedlings per plate, except for the outer two plates which were left empty. Inoculated and non-inoculated plants were provided weekly with $12 \mathrm{~L}$ of modified Hoagland solution containing the lower P levels $\left(7 \mu \mathrm{M} \mathrm{KH}_{2} \mathrm{PO}_{4}\right)$ as described previously. The full scheduling of nutrient supply and measurements is provided in Fig. 2.
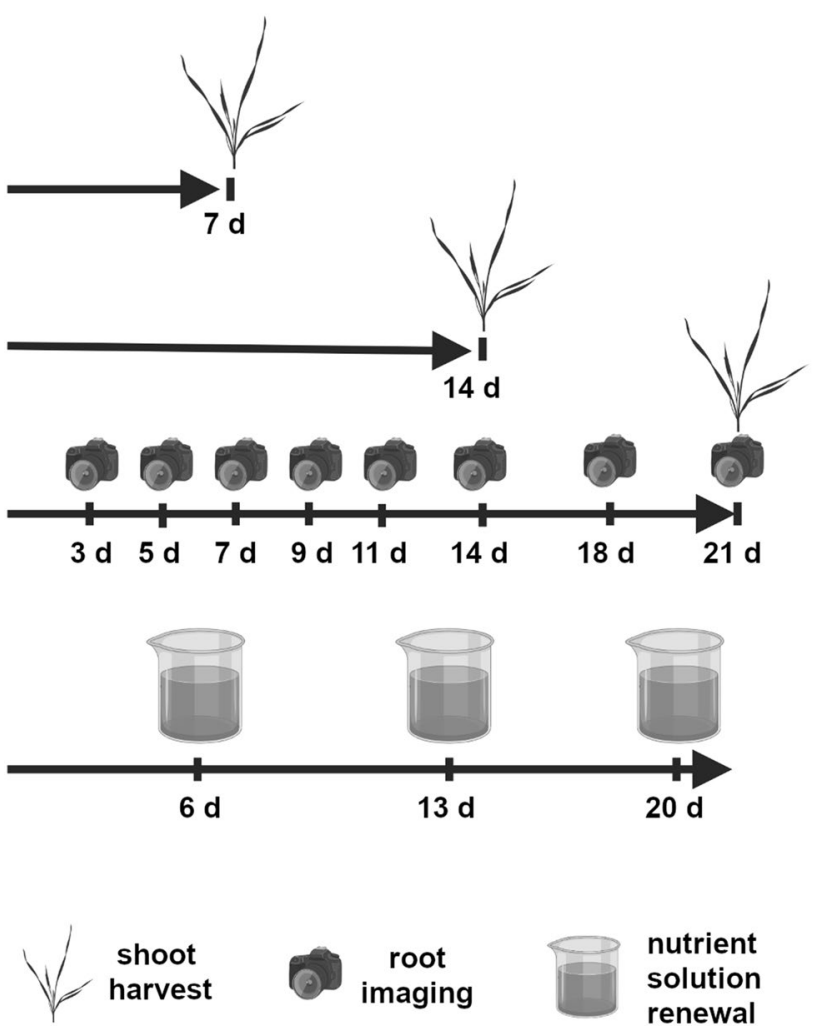

Fig. 2 Design and workflow of high throughput experiment to phenotype Brachypodium responses to Azospirillum in the GrowScreen$P a G e$ platform, which combined non-invasive with destructive measurements. The third timeline arrow shows the days that root systems of plants were imaged non-invasively (see Fig. 1d) after inoculation. These plants were harvested at 21 days for analysis of root fresh and dry weight and bacterial colonization by PCR (see Online resource 1), and shoot leaf area, shoot fresh and dry weight and nutrient analyses. Subgroups of plants were destructively harvested at 7, 14 days for leaf area, shoot fresh and dry weight and nutrient analyses. The nutrient solution in the GrowScreen-PaGe platform was replaced weekly 


\section{Shoot traits and nutrient analyses}

\section{First and second pilot experiment}

Inoculated and non-inoculated plants were harvested destructively at 21 days after inoculation (DAI). Roots were separated from the shoots with a scalpel and the leaf area was recorded with a leaf area meter (LI-COR Li3100, LI-COR Biosciences GmbH, Bad Homburg, DE). Shoots were then dried at $80{ }^{\circ} \mathrm{C}$ for $72 \mathrm{~h}$ and the shoot dry weight (DW) was measured using a digital scale with a $0.1 \mathrm{mg}$ resolution (Mettler Toledo, Columbus, USA).

\section{High throughput experiment}

Inoculated and non-inoculated plants were harvested destructively at 7, 14 and 21 DAI (Fig. 2) and leaf area and shoot DW were recorded as described above for each individual plant. After DW was determined, shoots of individual plants were pooled into three groups for each of the two treatments, each with approximately the same number of individuals. Pooled dry tissues were ground into a fine powder using a Retsch Mixer Mill MM400 (Retsch GmbH, Haan, DE) and analyzed for P, $\mathrm{N}$ and $\mathrm{K}$ elements. $\mathrm{N}$ was detected in an elemental analyzer (vario EL cube, Elementar, Langenselbold, DE) by combustion of $2 \mathrm{mg}$ dry shoot tissue and detection of their thermal conductivity, while $\mathrm{P}$ and $\mathrm{K}$ contents were recorded using microwave digestion and analysis by Inductively Coupled Plasma with Optical Emission Spectroscopy (ICP-OES) using $40 \mathrm{mg}$ dry shoot tissue. All nutrient analyses were performed at the Central Institute for Engineering, Electronics and Analytics (ZEA3 ) of Forschungszentrum Juelich (DE). The physiological P use efficiency (PPUE, expressed as $\frac{\operatorname{Shoot} D W}{\operatorname{Shoot}[P]}$ ) (Hammond et al. 2009) at each harvest point was recorded for both treatments.

\section{Root development analyses}

\section{First and second pilot experiment}

To non-destructively quantify root growth over time, roots were photographed at $3,5,7,9,11,14,18$ and 21 DAI using the image capturing box described in Gioia et al. (2016) equipped with 16 MP cameras that allow high resolution imaging ( $74 \mu \mathrm{m}$ per pixel) (Fig. 1d). Root images were analyzed from each plant using the PaintRhizo software package (Belachew et al. 2018; Nagel et al. 2012). At each time point, the total root length and root growth rate $\left(\right.$ root growth rate $=\frac{\left(\text { rootlengtht }_{1}-\text { rootlengtht }_{0}\right)}{t_{1}-t_{0}}$ were compared across plants, time and treatment.

\section{High throughput experiment}

As in the pilot experiments, roots were photographed at 3 , 5, 7, 9, 11, 14, 18 and 21 DAI (Fig. 2) and the length and growth rate of the whole root system were analyzed. To further understand when and how Azospirillum could alter the root systems, the PaintRhizo software was used to manually select and color the root types: axile roots (AR) and branch roots (BR) (Fig. 1d). Two criteria were used to separate AR and BR: the angle of growth and site of emergence. Axile roots developed vertically following gravity and came from either the seed (as in primary roots) or from the primary root if the primary root had stopped elongating close to the seed. Branch roots grew laterally (more horizontal to gravity) and developed from an AR (Roychoudhry and Kepinski 2015). In this experiment, no branch roots of an order higher than the first were observed.

\section{Validation of colonization by Azospirillum}

At 21 DAI in the pilot experiments and at 7, 14 and 21 DAI in the high throughput experiment, the roots of five plants per treatment were randomly chosen and PCR was used to validate that inoculated samples were colonized by Azospirillum, and that no cross-contamination occurred in noninoculated samples. The PCR used strain specific primers to amplify genomic DNA extracted from roots using an adapted version of the CTAB protocol from Doyle and Doyle (1987).

The PCR primers (FW 5' GGTGTTTCGCAACTCTCT GC 3', RV 5' GCACGACCTACACCTACTGG 3') were designed to amplify a $458 \mathrm{bp}$ strain specific portion of the A. brasilense Sp245 nitrite reductase sequence (Pothier et al. 2008) using the NCBI primer designing tool (Ye et al. 2012). Primers were synthesized by Eurofins Genomics $\mathrm{GmbH}$ (Ebersberg, DE). The PCR products were run on a $1 \%$ agarose gel to confirm amplification of target sequence.

Each PCR amplicon was sequenced by Sanger sequencing at LGC Genomics GmbH (Berlin, DE) and was compared against the NCBI database using the BLAST program (Altschul et al. 1990) to confirm sequence similarity to $A$. brasilense Sp245.

\section{Statistical analyses and data display}

The statistical significance of a difference between measured parameters in two treatments was analyzed using the Student's $t$-test (two tailed distribution, variance between treatments compared using Levene's test). Only those results with a significance level $(P$-value $)<0.05$ were 
considered reliable enough to reject the null hypothesis that the two treatments did not differ for a particular parameter. The difference between the two treatments, when significant, is displayed as a percentage $\left(\%\right.$ change $\left.=\frac{\text { parameter }_{\text {inoculated }} \text {-parameter } \text { non-inoculated }}{\text { parameter }_{\text {non-inoculated }}} \times 100\right)$.

\section{Results}

In the pilot and high throughput experiments in the GrowScreen-PaGe phenotyping system, Azospirillum influenced the above and below ground portions of plants. The DNA extraction, amplification with strain specific A. brasilense Sp245 specific primers and sequencing of the obtained amplicons confirmed that in the pilot experiments (data not shown) and at 7,14 and 21 DAI in the high throughput experiment (Online resource 1) bacteria-inoculated samples contained A. brasilense Sp245 DNA, while no products were amplified from DNA extracted from the non-inoculated samples.

\section{Shoot growth benefited from Azospirillum treatment}

\section{First pilot experiment}

Inoculated plants supplied with lower $\mathrm{P}\left(7 \mu \mathrm{M} \mathrm{KH} \mathrm{KHO}_{4}\right)$ had $17 \%$ greater leaf area at 21 DAI than inoculated plants provided with $25 \mu \mathrm{M} \mathrm{KH}_{2} \mathrm{PO}_{4}$. All other treatments did not differ (Online resource 2a). The shoot DW did not differ among the treatments (Online resource 3a).

\section{Second pilot experiment}

The repetition of the experiment with plants grown only with lower P provided different results from the first pilot experiment, as at 21 DAI inoculated plants had $36 \%$ greater leaf area compared to non-inoculated plants (Online resource $2 \mathrm{~b}$ ) and the shoot DW of inoculated plants was higher $(+21 \%)$ than non-inoculated plants (Online resource $3 b$ ).

\section{High throughput experiment}

The results from the high throughput experiment confirm those from the second pilot experiment. Leaf area was consistently higher in inoculated plants than non-inoculated plants, at levels of 16,13 and $14 \%$ at 7,14 and 21 DAI, respectively (Fig. 3a). This result was also reflected in the shoot DW, which was 12, 25 and 12\% greater in Azospirillum inoculated plants at those harvest points, respectively (Fig. 3b).

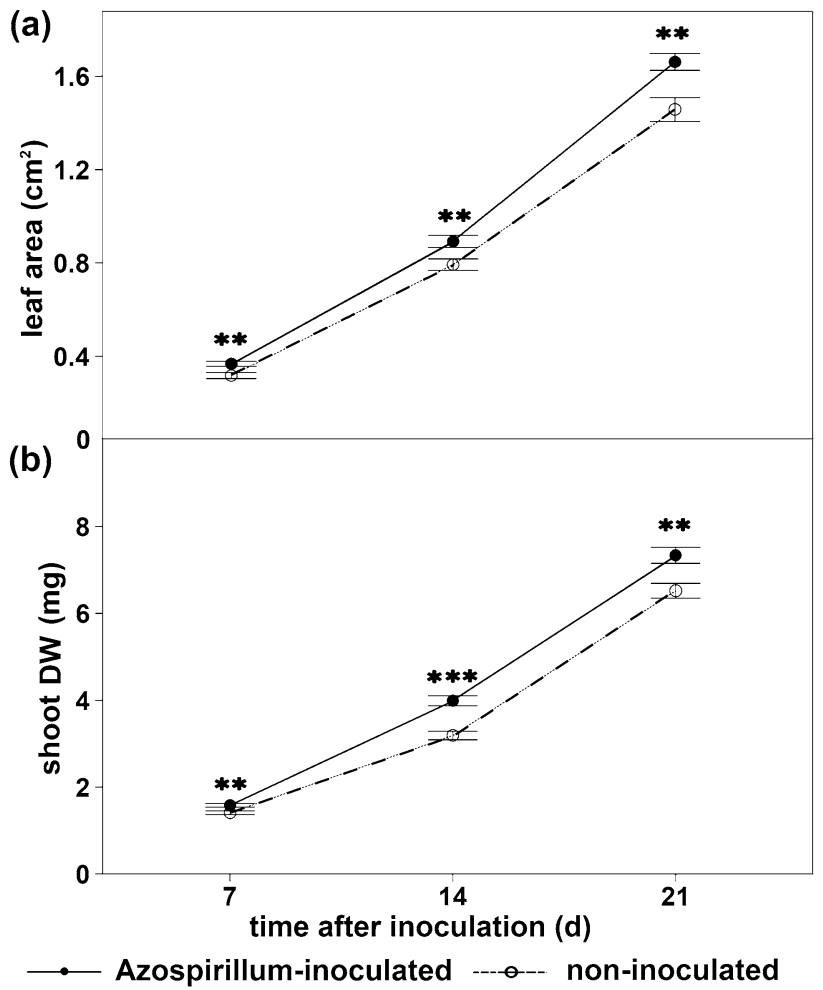

Fig. 3 Shoot growth of Brachypodium with or without Azospirillum inoculation and harvested after 7, 14 and 21 days in the GrowScreen-PaGe platform. (a) Leaf area. (b) Shoot dry weight (DW). Means \pm standard error presented. At 7 days, $n=87$ Azospirillum-inoculated and $n=87$ non-inoculated plants; 14 days, $n=25$ Azospirillum-inoculated and $n=37$ non-inoculated plants; 21 days, $n=28$ Azospirillum-inoculated and $n=32$ non-inoculated plants. Asterisks indicate probability of significant difference between mean of Azospirillum-inoculated and non-inoculated plants based on Student's t-test. $* p<0.05 ; * * p<0.01 ; * * * p<0.001$. DW dry weight

\section{The effect of inoculation with Azospirillum varied for different nutrients in Brachypodium shoots}

\section{High throughput experiment}

The concentration of $\mathrm{P}, \mathrm{N}$ and $\mathrm{K}$ in the shoots of inoculated and non-inoculated samples were similar at all harvest points (Fig. 4a, b, c), except that $\mathrm{N}$ at 14 DAI was $13.24 \%$ lower in inoculated plants than non-inoculated plants (Fig. 4b). The shoot concentration of $\mathrm{P}$ decreased approximately fourfold throughout the experiment in both treatments: at 7,14 and 21 DAI the $\mathrm{P}$ concentration was $3.61,1.88$ and $0.77 \mathrm{mg} \mathrm{g}^{-1}$ DW in inoculated plants and 3.90, 2.16 and $0.78 \mathrm{mg} \mathrm{g}^{-1} \mathrm{DW}$ in non-inoculated plants, respectively (Fig. 4a).

$\mathrm{P}$ content was also similar with or without bacterial treatment (Fig. 4d), while N (Fig. 4e) and K (Fig. 4f) contents were 15 and $17 \%$ greater, respectively, in plants treated with Azospirillum by 21 DAI. While N and K content increased steadily in both treatments throughout the 

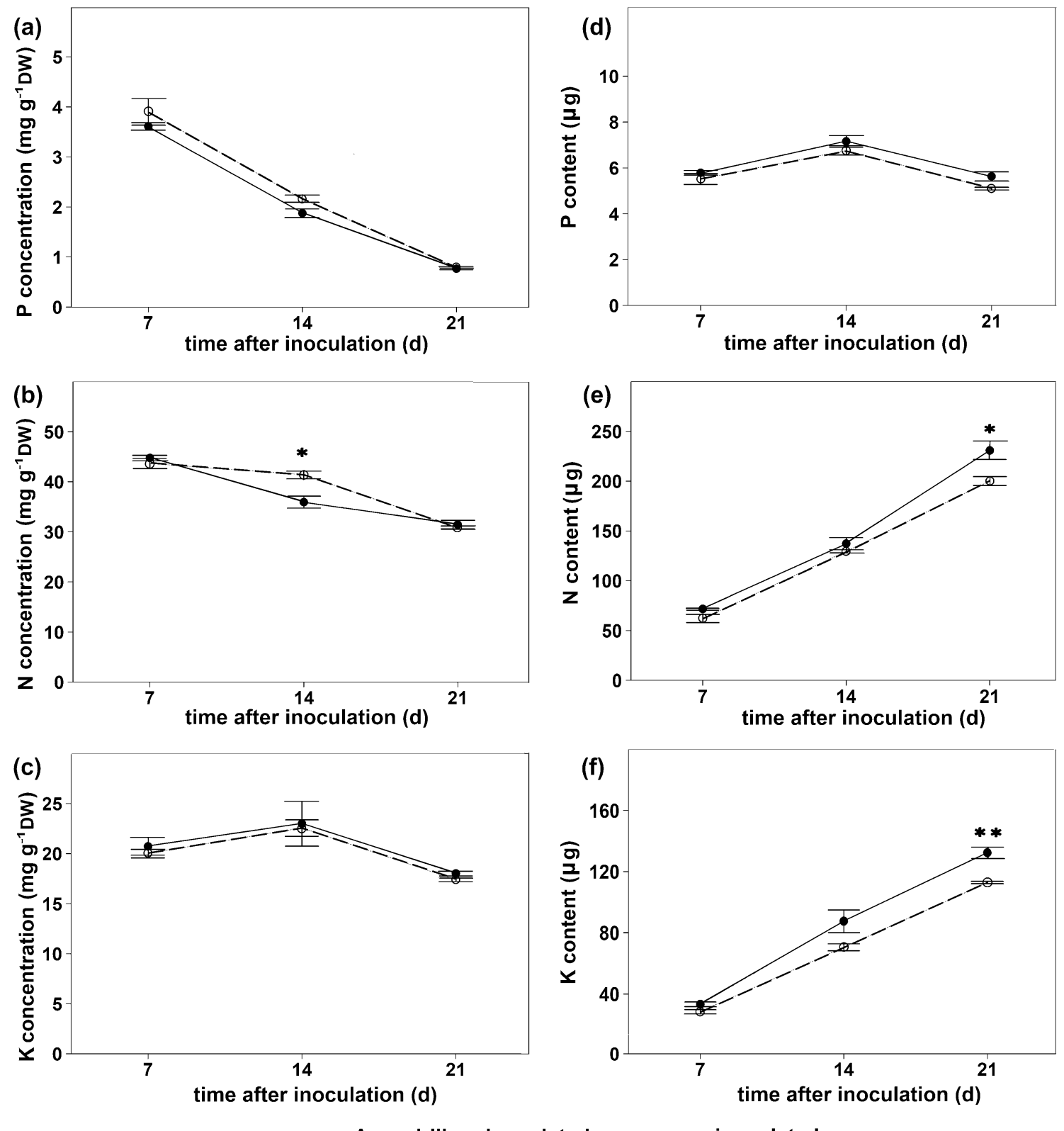

- Azospirillum-inoculated --- - non-inoculated

Fig. 4 Nutrients in Brachypodium shoots of plants with or without Azospirillum inoculation and harvested after 7, 14 and 21 days in the GrowScreen-PaGe platform. Concentration of phosphorus (a), nitrogen (b) and potassium (c), content of phosphorus (d), nitrogen (e) and potassium (f). All points are the mean \pm standard error of $n=3$ sam-

experiment, the shoot content of $\mathrm{P}$ remained around $6 \mu \mathrm{g}$ per shoot. The patterns of change of $\mathrm{P}, \mathrm{N}$ and $\mathrm{K}$ through the experiment are shown in Online resource 4.

Plants inoculated with Azospirillum had significantly higher physiological phosphorus use efficiency (PPUE; the shoot dry weight per P concentration in the shoot) than non-inoculated at 14 DAI (40\%) and 21 DAI (16\%) (Online resource 5). ples of plants pooled within each treatment. Asterisks indicate probability of significant difference between mean of A. brasilense-inoculated and non-inoculated plants based on Student's t-test. $* p<0.05$; $* * p<0.01 ; * * * p<0.001$

\section{Modulation of root development by Azospirillum at low $P$ depended on time after inoculation and type of root}

\section{First pilot experiment}

By 21 DAI, the root systems of plants inoculated with Azospirillum were $49 \%$ longer than those of non-inoculated 
plants in the lower $\mathrm{P}\left(7 \mu \mathrm{M} \mathrm{KH} \mathrm{KO}_{4}\right)$ condition. In contrast, inoculated and non-inoculated plants with higher $\mathrm{P}$ $\left(25 \mu \mathrm{M} \mathrm{KH}_{2} \mathrm{PO}_{4}\right.$ ) had similar root lengths (Online resource 6a). Inoculated plants supplied with lower $P$ had consistently longer roots and a higher root growth rate than non-inoculated plants grown at the same $\mathrm{P}$ concentration throughout the experiment (Online resource 6), and developed longer roots than plants grown at higher P levels after 14 DAI (Online resource 7). The difference in root length between inoculated plants grown in low $\mathrm{P}$ and those grown at higher $\mathrm{P}$ was detected between 11 and 14 DAI (Online resource $6,7)$.

\section{Second pilot experiment}

The second pilot experiment confirmed the low P treatment results from the first pilot experiment. At 21 DAI, inoculated plants had roots $21 \%$ longer than non-inoculated plants. The difference in the length of the root system in inoculated plants compared to non-inoculated plants became significant at 11 DAI (Online resource 8a). The length differences reflected growth rates, which were faster in inoculated plants between 7 and 11, and 11 and 14 DAI (69 and 72\%, respectively), but similar during the last week of the experiment (Online resource 8b).

\section{High throughput experiment}

As in the pilot experiments, inoculated plants in the high throughput experiment had significantly longer root systems than non-inoculated plants at 14 DAI (18\%), 18 DAI (33\%) and 21 DAI (32\%) (Fig. 5a). The differences in growth rate indicate greatest stimulation from inoculation took place between 14 and 18 DAI. Roots of inoculated plants elongated, on average, $27 \%$ faster than non-inoculated plants between 11 and $14 \mathrm{DAI} ; 47 \%$ faster between 14 and $18 \mathrm{DAI}$ and $31 \%$ faster between 18 and 21 DAI (Fig. 5d). Growth rates of roots of individual plants plotted in Online resource 9 highlight the distinct shift to faster root growth around 11 DAI in plants inoculated with Azospirillum.

Discriminating axile and branch roots revealed how inoculation specifically altered different root types (Fig. 5). Notably, the inoculated axile roots were shorter from 14 DAI $(-25,-32$ and $-36 \%$ at 14,18 and 21 DAI, Fig. $5 b)$ because they were growing slower from 7-9 DAI $(-28 \%$ between 7 and 9 DAI, $-29 \%$ between 9 and 11 DAI, $-46 \%$ between 11 and 14 DAI, $-44 \%$ between 14 and 18 DAI and $-48 \%$ between 18 and 21 DAI, Fig. 5e). In contrast, the branch roots of inoculated plants developed earlier and were consistently longer compared to non-inoculated plants. The first branch roots were observed in inoculated plants at 9 DAI, while non-inoculated plants started developing branch roots at $14 \mathrm{DAI}$, by which time branch roots of inoculated plants were more than fivefold longer (1.2 versus $8.4 \mathrm{~cm}$ in non-inoculated and inoculated plants). Inoculated plants had $199 \%$ longer branch roots at $18 \mathrm{DAI}$ and $133 \%$ longer branch roots at 21 DAI (Fig. 5c). Branch root growth rates were consistently higher in inoculated plants from 7-9 DAI (Fig. 5f), the time window when the growth rate of axile root in these plants started to slow.

Inoculated plants generally had longer roots than noninoculated plants irrespective of leaf area. The slope of the linear regression between those two parameters was steeper for inoculated plants, which were growing $72 \mathrm{~cm}$ root length per $\mathrm{cm}^{2}$ of leaf area while non-inoculated plants were growing $52 \mathrm{~cm}$ root length per $\mathrm{cm}^{2}$ leaf area (Online resource 10).

\section{Discussion}

These studies demonstrated that the PGP bacteria A. brasilense Sp245 stimulated the shoot and root growth of seedlings of the model grass $B$. distachyon $\mathrm{Bd} 21-3$ at suboptimal $\mathrm{P}$ and temperature conditions. Non-invasive phenotyping combined with destructive harvests resolved the following dynamics. First, inoculation altered shoot growth relative to root growth. Although plants had larger leaf area, the most noticeable difference was an increase in total root length which was $31 \%$ greater in inoculated compared to non-inoculated plants at 21 DAI. This result was mainly achieved by a higher root growth rate between 11 and 21 DAI (Fig. 5a, d, Online resource 9). Second, root types were differentially influenced by inoculation; inoculated plants had shorter axile roots, but their branch roots appeared earlier and were more developed than in non-inoculated plants (Fig. 5). We discuss these results in the context of shoot nutrients and propose a role for plant $\mathrm{P}$ in the timing and spatial effects on roots versus shoots.

\section{Azospirillum inoculation benefited shoot growth at low $\mathrm{P}$ and temperature regardless of root growth dynamics}

Shoots were more developed in inoculated than non-inoculated plants at all harvest time points (Fig. 3a, b), although roots were similar during the first half of the experiment. We propose that $\mathrm{P}$ levels in the plant modulated the positive effect of bacteria on roots in the second half of the experiment, while shoots responded to Azospirillum by improving plant tolerance to the cool temperature from the beginning of the experiment. Certain cold tolerant PGP bacteria, sourced from cold areas, have been reported to alleviate low temperature stress in cereals whereby inoculated plants showed increased shoot biomass, root growth, nutrient uptake, and water content (Mishra et al. 2011; Selvakumar et al. 2010; Yarzábal et al. 2018). These previous studies ascribe 

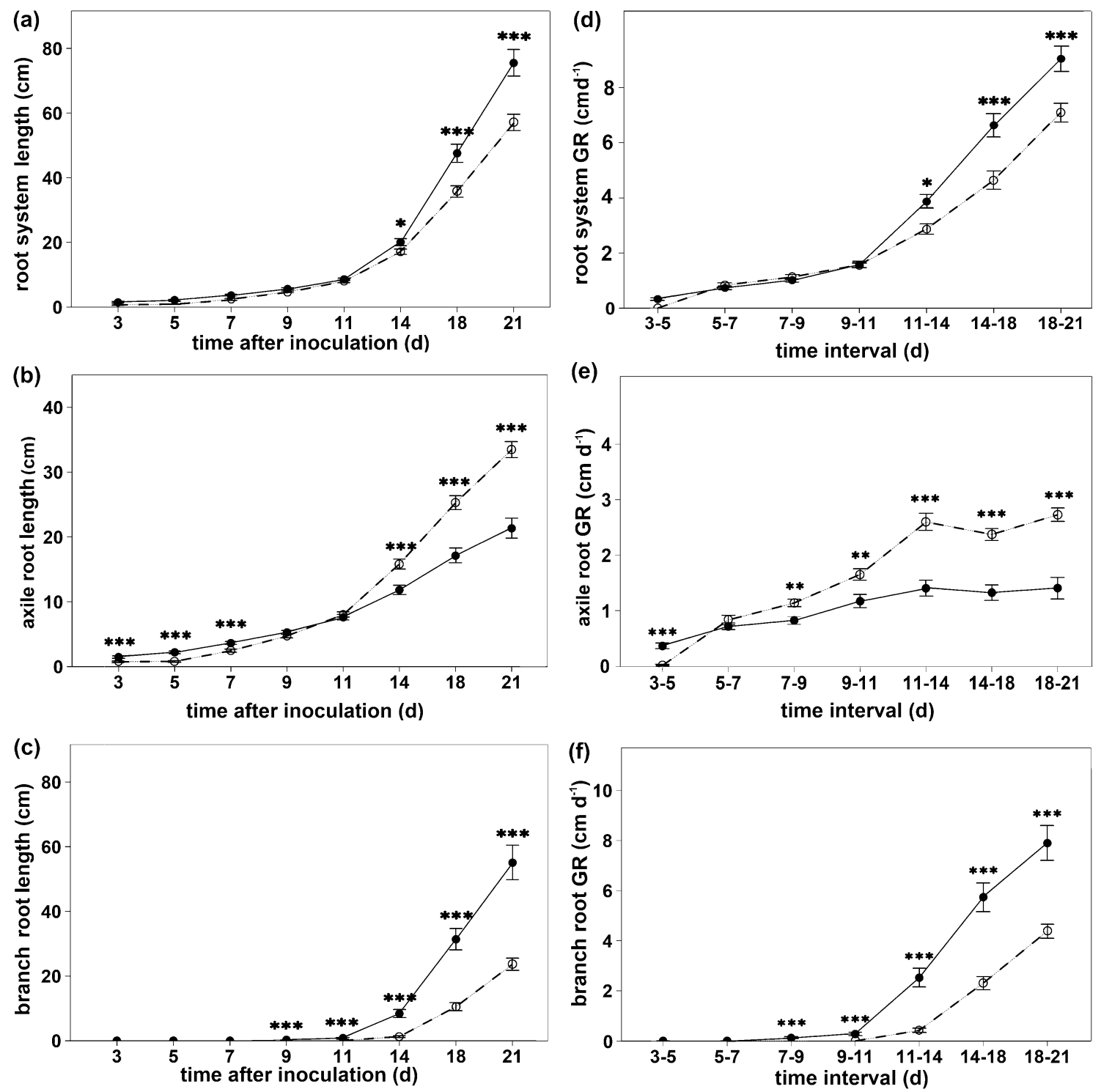

- Azospirillum-inoculated --a- non-inoculated

Fig. 5 Root growth of Brachypodium plants with or without Azospirillum inoculation and imaged non-destructively after 3, 5, 7, 9, $11,14,18$ and 21 days in the GrowScreen-PaGe platform. Images were analyzed with the PaintRhizo software. (a) Root system length. (b) Axile root length. (c) Branch root length. (d) Root system growth rate. (e) Axile root growth rate. (f) Branch root growth

improved acclimation to low temperatures to mechanisms such as the bacterial production of PGP hormones (mainly auxins), osmoprotectants that allowed osmotic adjustments for continued water uptake and enzymes capable of interrupting the biosynthesis of stress hormones in the plant. A further possible mechanism through which the bacterial treatment may have improved shoot development is the change in the concentration of cold-response proteins caused by tissue expansion in inoculated plants. Plant response to low temperature is regulated by a complex network of genes rate. Means \pm standard error presented. $n=28$ Azospirillum-inoculated and $n=32$ non-inoculated. Asterisks indicate probability of significant difference between mean of Azospirillum-inoculated and non-inoculated plants based on Student's t-test. $* p<0.05 ; * * p<0.01$; $* * * p<0.001$

and related transcription factors, whose tissue concentration can be affected by changes in plant growth rate (Zhao et al. 2020). Overall, additional biochemical and molecular studies are required with similar time resolved tissues to uncover hormone, signaling and omics changes underlying how Azospirillum stimulated Brachypodium shoot growth before root growth under low $\mathrm{P}$ and temperature conditions.

Interestingly, shoot growth was maintained in inoculated plants even though root growth increased. When leaf P concentration decreases, shoot carbohydrates can be reallocated, 
mainly in the form of sucrose, to the roots, where they function both as signaling molecules involved in rearrangement of root architecture and as a source of energy for those rearrangements (Hammond and White 2011). Low shoot $P$ levels can also decrease plant biomass by impairing photosynthesis. When the $\mathrm{P}$ reserves are depleted, this has direct effects on the energy transduction in thylakoids, inhibiting various Calvin cycle enzymes (Hammond and White 2008). In our study, inoculated plants showed not only a better developed root system, but also a higher shoot dry biomass and higher leaf area compared to non-inoculated plants, suggesting that the larger root system did not require redirection of resources needed for shoot development.

\section{Azospirillum inoculation did not increase shoot nutrient concentrations}

Shoot $\mathrm{P}$ concentration declined steadily through the experiment in inoculated and non-inoculated plants, due to shoot biomass increasing without an increase in $\mathrm{P}$ uptake. $\mathrm{P}$ is an essential element for early plant growth, and insufficient $\mathrm{P}$ availability can significantly alter plant physiology and development (Richardson et al. 2009b; White and Hammond 2008). As a general approximation, the critical leaf phosphorus concentration (the concentration in a diagnostic tissue that allows a crop to achieve $90 \%$ of its maximum yield) is $2-5 \mathrm{mg} \mathrm{g}^{-1} \mathrm{DW}$ (White and Brown 2010). The ICP analyses performed on Brachypodium shoots at 7, 14 and 21 DAI show that both inoculated and non-inoculated plants became $\mathrm{P}$ deficient in the second week of their growth and were suffering severe $\mathrm{P}$ deficiency at the end of the experiment (Fig. 4a). This result shows that the more developed root system of inoculated plants was unable to provide $\mathrm{P}$ to relieve tissue $\mathrm{P}$ deficiency.

Inoculation stimulated more shoot biomass per $\mathrm{P}$ and thus increased the PPUE (Online resource 5). This supports the hypothesis that $A$. brasilense $\mathrm{Sp} 245$ can increase the adaptation of grass plants to low $\mathrm{P}$ through their PPUE. A similar result was observed on wheat and corn inoculated with various PGP strains and grown on substrates with different P levels (Pereira et al. 2020; Talboys et al. 2014). Ours and these studies suggest that PGP bacteria can improve the performance of cereals in limiting $\mathrm{P}$ conditions also by improving plant PPUE. While the molecular mechanisms underlying this result need to be fully understood, the improved PPUE is of importance for agricultural environments where $\mathrm{P}$ is deficient.

The only difference in $\mathrm{N}$ shoot concentration was observed at $14 \mathrm{DAI}$, when it was higher in non-inoculated plants (Fig. 4b). There was a small and significant increase in $\mathrm{N}$ content at $21 \mathrm{DAI}$ (Fig. 4e), possibly due to stimulation of root elongation by Azospirillum. $\mathrm{N}$ fixation is probably the best known benefit that bacteria provide to legumes, but there is still a lack of experimental evidence that this is an important mechanism in interactions between cereals and bacteria (Dobbelaere et al. 1999; Rosenblueth et al. 2018). While there is one report of $A$. brasilense cd improving the growth of foxtail millet by fixing and providing $\mathrm{N}$ to inoculated plants supplied with suboptimal N levels (Kapulnik et al. 1981), most studies agree that the amount of $\mathrm{N}$ directly provided to cereals by $A$. brasilense strains is less significant than was previously hypothesized (Hungria et al. 2010; Rosenblueth et al. 2018). Our results seem to support the hypothesis that the beneficial role of Azospirillum on Brachypodium was not limited to diazotrophy, and was more related to promoting root length through branching.

Bacteria can enhance plant nutritional status by increasing the uptake of minerals from the soil, which can be done directly by converting those nutrients into more bioavailable forms, or indirectly by improving the development of plant roots, which then explore higher portions of soil (Shaharoona et al. 2008). In this experiment, shoots of inoculated plants had a higher $\mathrm{N}$ and $\mathrm{K}$ content per plant than non-inoculated plants (Fig. 4e, f), but this is probably a consequence of a more developed root system, that allowed exploration of a larger area, rather than the consequence of an active role of Azospirillum in nutrient uptake.

\section{Axile and branch roots were differentially affected by bacterial inoculation}

Thanks to their capacity to explore more portions of soil compared to axile roots, branch roots are particularly important in supporting plant growth in poor environments. Studies performed on barley (Nadira et al. 2016) and maize (Zhu and Lynch 2004) cultivars with varying performances at low $\mathrm{P}$ showed that the tolerant ones tend to conserve root branching, which improved $\mathrm{P}$ accumulation and relative growth rate compared to genotypes with less branch roots. We found here that Azospirillum inoculation enhanced root length specifically by enhancing branch root elongation. The root system of Brachypodium is highly responsive to $\mathrm{P}$ supply. In the first pilot experiment of this study, non-inoculated Brachypodium plants supplied with $7 \mu \mathrm{M}$ P had a significantly shorter root system than non-inoculated plants supplied with $25 \mu \mathrm{M}$ P (Online resource 7). A previous study of Brachypodium grown at varying $\mathrm{P}$ levels found that the growth of seminal roots was enhanced at moderately low $\mathrm{P}$ levels (Poiré et al. 2014). Interestingly, Ingram et al. (2012) found that different Brachypodium genotypes supplied with low $\mathrm{P}$ levels varied in root system length and branch root numbers. These studies did not distinguish between branch and axile roots. While we could not find previous studies on the effects of the interaction with any Azospirillum spp. on 
different root classes of Brachypodium, different A. brasilense strains have been observed to modify the root development of cereals, particularly that of branch roots. Malhotra and Srivastava (2009) inoculated sorghum, maize, wheat pearl millet and barley with A. brasilense SM and recorded a higher number of branch roots in sorghum and maize but not in barley and millet. In another study, pearl millet inoculated with A. brasilense Sp13t developed significantly longer branch roots than non-inoculated plants (Tien et al. 1979).

We propose that the bacterial treatment sustained branch root development particularly when plants started becoming $\mathrm{P}$ deficient, by stimulating earlier production of branch roots and increasing their growth rate during the second half of the experiment (Fig. 5). Development of branch roots can be a successful adaptation strategy in $P$ poor environments, as it may require lower $P$ investment per unit root length (Zhu and Lynch 2004). Root branching is typically increased by auxin accumulation, and the production of this hormone by bacteria is an important mechanism for their promotion of branch root growth (Goh et al. 2013). Phytohormones also play a decisive role in the rearrangements that occur in the root system of plants suffering P deficiency (Nadira et al. 2016; Talboys et al. 2014), and it is likely that bacterial PGP hormones can modify root responses in low P environments (Richardson et al. 2009a). Wheat plants inoculated with Bacillus amyloliquefaciens developed more biomass and longer roots, branch roots particularly, when grown at low P levels. The authors hypothesize that this result was linked to bacterial auxins, which increased root branching (Talboys et al. 2014).

As well as promoting branch root elongation, Azospirillum inoculation caused concomitant slowing of the parent axile roots. High auxin levels can also reduce root elongation, and it is possible that axile and branch root tips have different sensitivities to auxin from Azospirillum. Abiotic stresses such as soil strength (Watt et al. 2003) and salinity (Rahnama et al. 2011) cause axile root growth to slow, while branch root growth is stimulated. It was shown with modelling that a dynamic shift from axile to branch roots can reduce the energy costs of ATP-related transport substantially because membrane surface area is much less (Arsova et al. 2020). Our phenotyping study suggests that the interaction between Brachypodium and Azospirillum led to a rearrangement of the shoot and root system to allow the plant to respond efficiently to the low $\mathrm{P}$ and temperature conditions.

The GrowScreen-PaGe phenotyping platform is a promising tool to analyze the root growth of a large number of plants in response to PGP bacteria during various stages of their growth. However, the throughput and resolution of GrowScreen-PaGe did not allow us to extend to Brachypodium root hairs. Root hairs are important particularly in nutrient-poor environments (Nestler et al. 2016) and can play a significant role in the interaction with PGP bacteria (Mercado-Blanco and Prieto 2012). Brachypodium root hairs are sensitive to $\mathrm{P}$ concentrations and to compounds in soil solutions (Sasse et al. 2019). It would be very interesting to study if Azospirillum can alter the root hairs of cereals under low $\mathrm{P}$ and temperature conditions. While we hypothesize that in our study the bacteria improved plant adaptation to the combined abiotic stresses of low temperature and $\mathrm{P}$, further studies are needed to identify the mechanisms by which Azospirillum mediated growth changes, such as through a role in photosynthesis stimulation and reactive oxygen species (ROS) scavenging. Another limitation of the GrowScreen-PaGe is that this environment differs greatly from the one that plants and bacteria would be exposed to in the field. Although previous studies validating lab screens in agricultural fields have shown that germination-paper root screens robustly represent the root architecture of wheat grown in the field at the seedling stage (Rich et al. 2020; Watt et al. 2013), we are aware that the findings of our study need to be further validated in more realistic conditions. A great challenge for applying the results of bacteria-plant interaction studies to the agricultural sector is the transition from lab experiments (relatively small scale, controlled conditions, one or few stresses applied at the time) to "real" agricultural conditions, characterized by the presence of diverse microbiota in soils, constantly fluctuating growing conditions and multiple biotic interactions in both plants and PGP bacteria.

Our experiments show that Azospirillum can improve the shoot growth of the model plant Brachypodium in environments characterized by low temperature and this beneficial role extends to branch roots when plants are $\mathrm{P}$ deprived. The main phenotypic results observed in this study were larger inoculated shoots, which nevertheless had similar nutrient concentrations as non-inoculated plants, and longer branch roots at later stages of the experiments. The interactions between cereals and PGP bacteria in suboptimal environments are likely to become increasingly relevant in the coming decades. Our study allows resolution of beneficial developmental stages and time points to inform new management and genetic solutions for the use of PGP bacteria, thus contributing to a more economically and environmentally sustainable agriculture.

Acknowledgements The authors would like to acknowledge Anna Galinski, Carmen Müller, Kathrin Heinz, Jonas Lentz, Pablo Amador, Lucy Harrison, Carola Mohl and Tanja Ehrlich (IBG-2, Forschungszentrum Jülich, DE) for providing the necessary knowledge, technical help during harvest and some of the materials in the GrowScreen$\mathrm{PaGe}$ setup and for the introduction to the PaintRhizo software, Dr Volker Nischwitz and Michelle Hupert (ZEA-3, Forschungszentrum Jülich, DE) for the nutrient content analyses, Dr. Michael Rothballer (Helmholtz Zentrum München, Institute of Network Biology, Neuherberg, DE) for providing the A. brasilense Sp245 strain and Dr. Joelle 
Sasse and Dr. Trent Northen (Lawrence Berkeley National Laboratory, Berkley, USA) for providing the $B$. distachyon $\mathrm{Bd} 21-3$ seeds used in this study.

Funding Martino Schillaci is grateful for financial support from a University of Melbourne Research Scholarship provided through the Juelich Melbourne Postgraduate Academy (JUMPA). Michelle Watt holds the Adrienne Clarke Chair of Botany which is supported through the University of Melbourne Botany Foundation. Kerstin Nagel and Borjana Arsova acknowledge the Helmholtz Association of German Research Centres.

Data availability The datasets generated during and/or analyzed during the current study are available from the corresponding author on reasonable request.

\section{Complaince with ethical standards}

Conflict of interest All the authors of this manuscript declare that this study was conducted in the absence of any commercial or financial affiliation that could cause a conflict of interest.

Open Access This article is licensed under a Creative Commons Attribution 4.0 International License, which permits use, sharing, adaptation, distribution and reproduction in any medium or format, as long as you give appropriate credit to the original author(s) and the source, provide a link to the Creative Commons licence, and indicate if changes were made. The images or other third party material in this article are included in the article's Creative Commons licence, unless indicated otherwise in a credit line to the material. If material is not included in the article's Creative Commons licence and your intended use is not permitted by statutory regulation or exceeds the permitted use, you will need to obtain permission directly from the copyright holder. To view a copy of this licence, visit http://creativecommons.org/licenses/by/4.0/.

\section{References}

Altschul SF, Gish W, Miller W, Myers EW, Lipman DJ (1990) Basic local alignment search tool. J Mol Biol 215:403-410

Arsova B, Foster KJ, Shelden MC, Bramley H, Watt M (2020) Dynamics in plant roots and shoots minimize stress, save energy and maintain water and nutrient uptake. New Phytol 225:1111-1119

Baon JB, Smith SE, Alston AM (1994) Phosphorus uptake and growth of barley as affected by soil temperature and mycorrhizal infection. J Plant Nutr 17:479-492

Batten G, Wardlaw I, Aston M (1986) Growth and the distribution of phosphorus in wheat developed under various phosphorus and temperature regimes. Aust J Agric Res 37:459-469

Belachew KY, Nagel KA, Fiorani F, Stoddard FL (2018) Diversity in root growth responses to moisture deficit in young faba bean (Vicia faba L.) plants. PeerJ 6:e4401

Bertani G (1951) Studies on lysogenesis I.: the mode of phage liberation by lysogenic Escherichia coli. J Bacteriol 62:293

Biswas JC, Ladha JK, Dazzo FB, Yanni YG, Rolfe BG (2000) Rhizobial inoculation influences seedling vigor and yield of rice. Agron J 92:880-886

Casanovas EM, Barassi CA, Sueldo RJ (2002) Azospirillum inoculation mitigates water stress effects in maize seedlings. Cereal Res Commun 30:343-350

Catalan P et al. (2014) Update on the genomics and basic biology of brachypodium: international brachypodium initiative (IBI). Trends Plant Sci 19:414-418
Cordell D, Rosemarin A, Schröder JJ, Smit A (2011) Towards global phosphorus security: a systems framework for phosphorus recovery and reuse options. Chemosphere 84:747-758

Fonseca Bredada FA, Silvada TFR, Santosdos SG, Alves GC, Reis VM (2019) Modulation of nitrogen metabolism of maize plants inoculated with Azospirillum brasilense and Herbaspirillum seropedicae. Arch Microbol 201:558

Delaplace P, et al. (2015) Influence of rhizobacterial volatiles on the root system architecture and the production and allocation of biomass in the model grass Brachypodium distachyon (L.) P. Beauv. BMC Plant Biol 15:195

Dhillon J, Torres G, Driver E, Figueiredo B, Raun WR (2017) World phosphorus use efficiency in cereal crops. Agron J 109:1670-1677

Do Amaral FP, Pankievicz VC, Arisi ACM, de Souza EM, Pedrosa F, Stacey G (2016) Differential growth responses of Brachypodium distachyon genotypes to inoculation with plant growth promoting rhizobacteria. Plant Mol Biol 90:689-697

Dobbelaere S, Croonenborghs A, Thys A, Broek AV, Vanderleyden J (1999) Phytostimulatory effect of Azospirillum brasilense wild type and mutant strains altered in IAA production on wheat. Plant Soil 212:153-162

Doyle JJ, Doyle JL (1987) A rapid DNA isolation procedure for small quantities of fresh leaf tissue. Phytochem Bull 19:11-15

Egamberdieva D (2009) Alleviation of salt stress by plant growth regulators and IAA producing bacteria in wheat. Acta Physiol Plant $31: 861-864$

FAO (2018) World Food and Agriculture - Statistical Pocketbook 2018. FAO, Rome, p 254

Fitzgerald TL et al (2015) Brachypodium as an emerging model for cereal-pathogen interactions. Ann Bot 115:717-731

Flavel RJ, Guppy CN, Tighe M, Watt M, McNeill A, Young IM (2012) Non-destructive quantification of cereal roots in soil using highresolution X-ray tomography. J Exp Bot 63:2503-2511

Gagne-Bourque F, Mayer BF, Charron J-B, Vali H, Bertrand A, Jabaji $S$ (2015) Accelerated growth rate and increased drought stress resilience of the model grass Brachypodium distachyon colonized by Bacillus subtilis B26. PLoS ONE 23(10):e0130456

Gholami A, Shahsavani S, Nezarat S (2009) The effect of plant growth promoting rhizobacteria (PGPR) on germination, seedling growth and yield of maize. Int J Agric Biosyst Eng 3:35-40

Gioia $\mathrm{T}$ et al (2016) GrowScreen-PaGe, a non-invasive, high-throughput phenotyping system based on germination paper to quantify crop phenotypic diversity and plasticity of root traits under varying nutrient supply. Funct Plant Biol 44:76-93

Goh C-H, Vallejos DFV, Nicotra AB, Mathesius U (2013) The impact of beneficial plant-associated microbes on plant phenotypic plasticity. J Chem Ecol 39:826-839

Grant C, Flaten D, Tomasiewicz D, Sheppard S (2001) The importance of early season phosphorus nutrition. Can J Plant Sci 81:211-224

Hammond JP et al (2009) Shoot yield drives phosphorus use efficiency in Brassica oleracea and correlates with root architecture traits. J Exp Bot 60:1953-1968

Hammond JP, White PJ (2008) Sucrose transport in the phloem: integrating root responses to phosphorus starvation. J Exp Bot 59:93-109

Hammond JP, White PJ (2011) Sugar signaling in root responses to low phosphorus availability. Plant Physiol 156:1033-1040

Hanway J, Olson R (1980) Phosphate nutrition of corn, sorghum, soybeans, and small grains. In: The role of phosphorus in agriculture. Wiley, Amsterdam, p 681-692

Harsant J, Pavlovic L, Chiu G, Sultmanis S, Sage TL (2013) High temperature stress and its effect on pollen development and morphological components of harvest index in the $\mathrm{C} 3$ model grass Brachypodium distachyon. J Exp Bot 64:2971-2983 
Hoagland DR, Arnon DI (1950) The water-culture method for growing plants without soil. Circ Calif Agric Exp Stn 347:32

Hungria M, Campo RJ, Souza EM, Pedrosa FO (2010) Inoculation with selected strains of Azospirillum brasilense and A. lipoferum improves yields of maize and wheat in Brazil. Plant Soil 331:413-425

Ingram PA, Zhu J, Shariff A, Davis IW, Benfey PN, Elich T (2012) High-throughput imaging and analysis of root system architecture in Brachypodium distachyon under differential nutrient availability. Philos Trans R Soc B: Bio Sci 367:1559-1569

Kapulnik Y, Okon Y, Kigel J, Nur I, Henis Y (1981) Effects of temperature, nitrogen fertilization, and plant age on nitrogen fixation by Setaria italica inoculated with Azospirillum brasilense (strain cd). Plant Physiol 68:340-343

Malhotra M, Srivastava S (2009) Stress-responsive indole-3-acetic acid biosynthesis by Azospirillum brasilense SM and its ability to modulate plant growth. Eur J Soil Biol 45:73-80

McMichael B, Quisenberry J (1993) The impact of the soil environment on the growth of root systems. Environ Exp Bot 33:53-61

Mercado-Blanco J, Prieto P (2012) Bacterial endophytes and root hairs. Plant Soil 361:301-306

Mishra PK et al (2011) Alleviation of cold stress in inoculated wheat (Triticum aestivum L.) seedlings with psychrotolerant Pseudomonads from NW Himalayas. Arch Microbiol 193:497-513

Nadira UA, Ahmed IM, Wu F, Zhang G (2016) The regulation of root growth in response to phosphorus deficiency mediated by phytohormones in a Tibetan wild barley accession. Acta Physiol Plant 38:105

Nagel KA et al (2012) GROWSCREEN-Rhizo is a novel phenotyping robot enabling simultaneous measurements of root and shoot growth for plants grown in soil-filled rhizotrons. Funct Plant Biol 39:891-904

Nestler J, Keyes SD, Wissuwa M (2016) Root hair formation in rice (Oryza sativa L.) differs between root types and is altered in artificial growth conditions. J Exp Bot 67:3699-3708

Palta J, Watt M (2009) Vigorous crop root systems: form and function for improving the capture of water and nutrients. Applied crop physiology: boundaries between genetic improvement and agronomy. Academic Press, San Diego, pp 309-325

Pereira NCM, Galindo FS, Gazola RPD, Dupas E, Rosa PAL, Mortinho ES (2020) Corn Yield and Phosphorus Use Efficiency Response to Phosphorus Rates Associated With Plant Growth Promoting Bacteria. Front Environ Sci 8:40

Pereyra MA, Ballesteros FM, Creus CM, Sueldo RJ, Barassi CA (2009) Seedlings growth promotion by Azospirillum brasilense under normal and drought conditions remains unaltered in Tebuconazole-treated wheat seeds. Eur J Soil Biol 45:20-27

Pflugfelder D, Metzner R, van Dusschoten D, Reichel R, Jahnke S, Koller R (2017) Non-invasive imaging of plant roots in different soils using magnetic resonance imaging (MRI). Plant Methods $13: 102$

Poiré R, Chochois V, Sirault XR, Vogel JP, Watt M, Furbank RT (2014) Digital imaging approaches for phenotyping whole plant nitrogen and phosphorus response in Brachypodium distachyon. J Integr Plant Biol 56:781-796

Pothier JF, Prigent-Combaret C, Haurat J, Moënne-Loccoz Y, Wisniewski-Dyé F (2008) Duplication of plasmid-borne nitrite reductase gene nirK in the wheat-associated plant growth-promoting rhizobacterium Azospirillum brasilense Sp245. Mol Plant-Microbe Interact 21:831-842

Rahnama A, Munns R, Poustini K, Watt M (2011) A screening method to identify genetic variation in root growth response to a salinity gradient. J Exp Bot 62:69-77

Rich SM, Christopher J, Richards R, Watt M (2020) Root phenotypes of young wheat plants grown in controlled environments show inconsistent correlation with mature root traits in the field. J Exp Bot 71:4751-4762

Richards R, Watt M, Rebetzke G (2007) Physiological traits and cereal germplasm for sustainable agricultural systems. Euphytica 154:409-425

Richardson AE, Barea J-M, McNeill AM, Prigent-Combaret C (2009) Acquisition of phosphorus and nitrogen in the rhizosphere and plant growth promotion by microorganisms. Plant Soil 321:305-339

Richardson AE, Hocking PJ, Simpson RJ, George TS (2009) Plant mechanisms to optimise access to soil phosphorus. Crop Pasture Sci 60:124-143

Rosenblueth M et al (2018) Nitrogen fixation in cereals. Frontiers Microbiol 9:1794-1800

Rothballer M, Schmid M, Fekete A, Hartmann A (2005) Comparative in situ analysis of ipdC-gfpmut 3 promoter fusions of Azospirillum brasilense strains Sp7 and Sp245. Environ Microbiol 7:1839-1846

Roychoudhry S, Kepinski S (2015) Shoot and root branch growth angle control-the wonderfulness of lateralness. Curr Opin Plant Biol 23:124-131

Sasse J et al (2019) Multilab EcoFAB study shows highly reproducible physiology and depletion of soil metabolites by a model grass. New Phytol 222:1149-1160

Schneebeli K, Mathesius U, Zwart AB, Bragg JN, Vogel JP, Watt M (2016) Brachypodium distachyon genotypes vary in resistance to Rhizoctonia solani AG8. Funct Plant Biol 43:189-198

Selvakumar G, Kundu S, Joshi P, Nazim S, Gupta A, Gupta H (2010) Growth promotion of wheat seedlings by Exiguobacterium acetylicum 1P (MTCC 8707) a cold tolerant bacterial strain from the Uttarakhand Himalayas. Indian J Microbiol 50:50-56

Shaharoona B, Naveed M, Arshad M, Zahir ZA (2008) Fertilizerdependent efficiency of Pseudomonads for improving growth, yield, and nutrient use efficiency of wheat (Triticum aestivum L). Appl Microbiol Biot 79:147-155

Talboys PJ, Owen DW, Healey JR, Withers PJ, Jones DL (2014) Auxin secretion by Bacillus amyloliquefaciens FZB42 both stimulates root exudation and limits phosphorus uptake in Triticum aestivum. BMC Plant Biol 14:51

Tien T, Gaskins M, Hubbell D (1979) Plant growth substances produced by Azospirillum brasilense and their effect on the growth of pearl millet (Pennisetum americanum L.). Appl Environ Microbiol 37:1016-1024

Wasson AP, Nagel KA, Tracy S, Watt M (2020) Beyond digging: noninvasive root and rhizosphere phenotyping. Trends Plant Sci 25:119-120

Watt M, McCully ME, Kirkegaard JA (2003) Soil strength and rate of root elongation alter the accumulation of Pseudomonas spp. and other bacteria in the rhizosphere of wheat. Funct Plant Biol 30:483-491

Watt M, Moosavi S, Cunningham SC, Kirkegaard J, Rebetzke G, Richards R (2013) A rapid, controlled-environment seedling root screen for wheat correlates well with rooting depths at vegetative, but not reproductive, stages at two field sites. Ann Bot 112:447-455

White P, Brown P (2010) Plant nutrition for sustainable development and global health. Ann Bot 105:1073-1080

White PJ, Hammond JP (2008) Phosphorus nutrition of terrestrial plants. The ecophysiology of plant-phosphorus interactions. Springer, Dordrecht, pp 51-81

Wintermans PC, Bakker PA, Pieterse CM (2016) Natural genetic variation in Arabidopsis for responsiveness to plant growthpromoting rhizobacteria. Plant Mol Biol 90:623-634

Yarzábal LA, Monserrate L, Buela L, Chica E (2018) Antarctic Pseudomonas spp. promote wheat germination and growth at low temperatures. Polar Biol 41:2343-2354 
Ye J, Coulouris G, Zaretskaya I, Cutcutache I, Rozen S, Madden TL (2012) Primer-BLAST: a tool to design target-specific primers for polymerase chain reaction. BMC Bioinf 13:134

Zhang C et al (2018) Do longer root hairs improve phosphorus uptake? Testing the hypothesis with transgenic Brachypodium distachyon lines overexpressing endogenous RSL genes. New Phytol 217:1654-1666

Zhao Y, Antoniou-Kourounioti RL, Calder G, Dean C, Howard M (2020) Temperature-dependent growth contributes to long-term cold sensing. Nature 583:825-829
Zhu J, Lynch JP (2004) The contribution of lateral rooting to phosphorus acquisition efficiency in maize (Zea mays) seedlings. Funct Plant Biol 31:949-958

Publisher's Note Springer Nature remains neutral with regard to jurisdictional claims in published maps and institutional affiliations. 